\title{
L'agriculture tunisienne face à la Covid-19: impacts de la crise sanitaire et perspectives pour une agriculture résiliente
}

\author{
Mohamed Elloumi* \\ Université de Carthage, INRAT, LR16INRAT07, Laboratoire d'économie rurale, rue Hédi Karray, 1004 El Menzah, Tunisie
}

\begin{abstract}
Résumé - L'économie tunisienne a subi de plein fouet les effets de la crise sanitaire Covid-19 et des mesures indispensables pour la juguler prises par les pouvoirs publics comme la fermeture des frontières et le confinement de la population. L'agriculture a dû faire face aux mêmes difficultés; toutefois, la courte durée du confinement total et la décision des autorités d'exclure les activités agricoles de certaines restrictions ont réduit les effets de la crise sanitaire sur la disponibilité des produits alimentaires. Cependant, la baisse du pouvoir d'achat des franges les plus vulnérables de la population a réduit leur accès à certains produits de base. En définitive, ce sont les principales filières agricoles tunisiennes d'exportation (dattes, huile d'olive...) qui ont été les plus impactées du fait de la baisse de la demande internationale. Enfin, si la réponse des agriculteurs et des différents acteurs qui encadrent le secteur a permis une certaine résilience, il n'en demeure pas moins que cette conjoncture a servi de révélateur d'une crise plus profonde du système alimentaire et agricole qui doit faire face à des défis majeurs comme la dépendance aux marchés extérieurs pour plusieurs produits de base (céréales, soja, viande rouge, huile de graine...) et dont le dépassement nécessite une refonte à la fois du modèle technique et du pacte social entre agriculteurs et consommateurs.
\end{abstract}

Mots clés : politique agricole tunisienne / Covid-19 / sécurité alimentaire / marché international

\begin{abstract}
Tunisian agriculture in the face of Covid-19: impacts of the health crisis and prospects for a resilient agriculture. Tunisian economy has been strongly affected by the Covid-19 health crisis and by the government's response, closing borders and confining population. The agricultural sector in its turn has faced the same difficulties; however, the short-lived lockdown and the authorities' decision to exempt agricultural activities from some restrictions reduced the effects on products availability. However, drop in the purchasing power of the population's most vulnerable groups reduced their access to some basic products. In the end, it was the decrease of international demand that affected most Tunisia's main agricultural sectors. While the response of farmers and various actors supervising the agricultural activities resulted in a certain resilience of the sector, the fact nevertheless remains that this situation revealed a deeper crisis in agriculture which is facing major challenges; overcoming them requires an overhaul of both the technical model and the social pact between farmers and consumers.
\end{abstract}

Keywords: Tunisian agricultural policy / Covid-19 / food security / international market

\section{1 introduction}

La crise sanitaire causée par la Covid-19 a impacté l'économie tunisienne comme l'économie mondiale. Fortement ouverte sur le reste du monde, l'économie tunisienne a dû faire face au quasi-arrêt de l'appareil de production (industrie automobile), au ralentissement de la demande en produits d'exportation et à l'arrêt de certains services comme le

\footnotetext{
* Auteur de correspondance :

elloumi.mohamed@iresa.agrinet.tn
}

transport aérien, et surtout d'une activité économique dominante en Tunisie, le tourisme. D'autres secteurs ont aussi été fortement impactés, tels que le bâtiment touché par l'arrêt de nombreux chantiers, ou le secteur du transport des marchandises et des personnes qui a été contraint de réduire fortement ses activités par manque de clients et du fait de l'interdiction des déplacements entre les régions

Au niveau mondial, le confinement imposé à la population avec plus ou moins de sévérité dans plusieurs pays a affecté le fonctionnement de l'agriculture et des systèmes alimentaires du fait des perturbations dans les processus de production et de transformation, et le transport des produits vers les centres de 
consommation. La crise a aussi agi sur la demande en produits agricoles du fait de la fermeture des restaurants et surtout de l'arrêt du tourisme international et de la réduction des échanges internationaux. Par contre, la crise a faiblement impacté l'offre de produits agricoles vivriers, même dans les grands centres urbains tunisiens (FAO, 2020a, 2020b).

Cet article vise à comprendre les effets de la crise sanitaire sur l'agriculture et les systèmes alimentaires tunisiens et les capacités d'adaptation des agriculteurs et des autres acteurs de ce secteur. Pour cela, nous avons mobilisé des données récentes du ministère de l'Agriculture et de l'Observatoire national de l'agriculture (ONAGRI: www.onagri.nat.tn), les études de l'Institut national de la statistique (INS: www.ins.tn) et de la Banque mondiale relatives à l'impact de la crise et notamment du confinement sur les ménages (INS et Banque mondiale, 2020a, 2020b), celles sur la pauvreté et la précarité des ménages durant cette crise (Mahjoub, 2020) et, enfin, l'étude menée par une équipe IFPRI et IEQC (International Food Policy Research Institute; Institut tunisien de la compétitivité et des études quantitatives) simulant les impacts à venir de la crise sur les performances des différents secteurs de l'économie selon la durée du confinement (Elkadhi et al., 2020).

En parallèle, nous avons réalisé une enquête en ligne (du $1^{\text {er }}$ au 15 juin 2020) auprès de 30 agriculteurs, responsables de syndicats et/ou d'associations de producteurs portant sur leur perception de la situation économique suite au développement de la pandémie en Tunisie, leurs stratégies d'adaptation et les réponses qu'ils pensent souhaitables d'apporter à une telle crise.

Le questionnaire visait principalement à orienter un entretien ouvert sur les conséquences du confinement sur le fonctionnement de l'agriculture en général et de leur exploitation en particulier, sur l'accès aux intrants et la commercialisation de leur production et en définitive, sur leur revenu actuel comparé à celui de l'année précédente.

Malgré le faible nombre de personnes interrogées, la qualité de certains interlocuteurs (responsables syndicaux au niveau national et régional, agriculteurs de différentes régions du pays et avec différentes activités, etc.) nous a permis de bien contextualiser notre travail de synthèse.

Nous aborderons, dans un premier temps, les effets déjà constatés de la Covid-19 sur l'agriculture tunisienne, ensuite nous traiterons de l'effet de la crise sanitaire sur la demande nationale et internationale en produits agricoles tunisiens et, en fin d'article, nous discuterons des leçons à tirer de cette crise, notamment celles en rapport avec le modèle de développement agricole et la place de l'agriculture dans l'économie nationale.

\section{Les répercussions de la crise sur la production agricole}

Selon l'OMS, la Tunisie a atteint le stade 3 de la crise sanitaire le 23 mars 2020 avec de multiples foyers de contamination qui pouvaient menacer le bon fonctionnement du système de santé, affaibli depuis les années 1990 par les suites du programme d'ajustement structurel.

Face à ce risque, les pouvoirs publics ont décrété, le 18 mars, un couvre-feu de $18 \mathrm{~h}$ à $6 \mathrm{~h}$ du matin et, le 23 mars, le confinement total de la population et la fermeture des frontières. La levée des mesures restrictives s'est faite en plusieurs phases: déconfinement le 4 mai, autorisation des déplacements entre régions à partir du 4 juin, levée du couvrefeu le 8 juin et, enfin, le 27 juin, réouverture des frontières. Ces mesures ont eu des effets négatifs sur les secteurs de l'économie tunisienne dépendant des transports internationaux et nationaux, et en particulier le tourisme.

Ainsi, l'impact total du confinement correspond à une réduction du Produit intérieur brut (PIB) de près de $50 \%$ au second trimestre 2020. Par ailleurs, le Gouvernement tunisien estime que l'année 2020 connaîtra une croissance négative de $-6 \%$, la perte d'environ 250000 emplois et un taux de chômage de $20 \%$ de la population active, alors qu'il était de $15,1 \%$ avant la crise.

\subsection{De faibles perturbations dans les processus de production}

Les résultats de notre enquête en ligne concernant la production ne montrent pas de grandes perturbations dans la conduite des cultures et de l'élevage durant le confinement, en premier lieu, du fait que les agriculteurs pouvaient poursuivre leurs travaux comme avant.

Concernant l'élevage, et vu la forte dépendance de ce secteur par rapport aux importations des matières constituant les aliments composés, le gouvernement a décidé d'exempter de confinement le personnel des usines d'aliment du bétail et d'accorder la libre circulation de ce type d'intrant dans tout le pays. Ces mesures ont permis un approvisionnement assez régulier des points de vente et des élevages. De plus, la période de confinement correspondait au printemps, période marquée par une disponibilité élevée en ressources pastorales et fourragères, ce qui a permis, selon un responsable syndical, la poursuite de l'alimentation du cheptel sans encombre.

La production de lait bovin n'a pas rencontré de problème majeur car tous les acteurs de l'aval se sont mobilisés pour assurer une collecte régulière du lait, sa transformation, puis sa distribution dans tout le pays.

Toutefois, l'impact du confinement sur la production pourrait être observé plus tard, car les cultures qui devaient être semées pendant le confinement ou qui ont eu besoin de soins particuliers durant cette période ont pu être retardées ou affectées dans leur développement, ce qui causera des baisses de production quelques mois plus tard. C'est le cas, par exemple, des cultures maraîchères d'été, semées en mars avril, et de la pollinisation des palmiers dattiers, réalisée à la même période.

Le secteur stratégique de l'huile d'olive n'a pas connu de grandes perturbations en termes de production car la fin de la récolte n'a pas été affectée, mais des difficultés ont été signalées en ce qui concerne la taille des oliviers du fait des restrictions de déplacement de la main-d'œuvre.

Quant à la production des céréales, la période des moissons a démarré le 4 juin, donc en période de confinement «ciblé », et, de ce fait, n'a pas été impactée. Mais les prévisions annoncent une récolte médiocre de seulement 15,7 millions de quintaux, en baisse d'environ $30 \%$ par rapport à celle de l'année précédente. Ce qui impliquera pour la Tunisie un besoin d'approvisionnement sur les marchés internationaux d'au moins 15 millions de quintaux de céréales diverses.

Selon nos enquêtes, le manque de disponibilité des pièces de rechange et de certains intrants (pesticides, semences) a 
affecté l'exécution de certaines opérations culturales chez certains agriculteurs mais sans entraîner une baisse significative de la production nationale.

En définitive, ce sont les petites exploitations qui ont le plus souffert du confinement du fait de leur faible capacité d'adaptation due à une trésorerie/épargne limitée et de leur mode d'approvisionnement et de mise en marché dépendant des souks hebdomadaires qui ont été fermés dans plusieurs régions du pays dès les premiers jours du confinement. À cela s'ajoute le manque d'emplois occasionnels pour les actifs de ces petites exploitations, sachant que plus de $40 \%$ des chefs d'exploitation tunisiens sont pluriactifs et ont donc besoin d'une activité rémunératrice hors de leur exploitation pour faire vivre leur famille.

À l'inverse, les grandes exploitations, mieux connectées aux marchés (marchés de gros, centrales d'achat et même e-commerce, qui ont fonctionné presque normalement) ne semblent pas avoir beaucoup souffert du confinement.

\subsection{Contraintes de commercialisation et impact sur l'offre de produits agricoles}

Globalement, la période du confinement n'a pas été caractérisée par une tension forte entre offre et demande de produits alimentaires en Tunisie. La baisse de l'offre a été relativement modérée et dans tous les cas, elle a pu répondre à la demande qui a connu à son tour une certaine contraction. L'existence de stocks de régulation (céréales, huiles, sucre) et l'arrivée à maturité de nombreuses productions (pommes de terre, céréales) pendant la période de crise a permis de maintenir l'offre de ces produits.

Pour les cultures maraîchères et arboricoles, certaines productions consommées en frais ont connu des difficultés d'écoulement du fait des perturbations dans les circuits de distribution. C'est le cas de la pomme de terre qui a souffert de la fermeture (i) de la restauration collective, lieu de consommation de la plus grande quantité de ce produit et (ii) des frontières terrestres avec la Lybie, un des principaux marchés pour les produits agricoles tunisiens; ainsi, les exportations de fruits et légumes vers ce marché ont connu une baisse d'environ $22 \%$ par rapport à la même période de l'année précédente (ONAGRI, 2020a, 2020b). Dans le cas de la pomme de terre, l'État a mobilisé un fonds de 5 millions de dinars tunisiens - DT (1,5 millions d'euros) pour subventionner le recours au stockage. Il a été observé une baisse des apports d'agrumes (en fin de récolte) et de fruits à noyaux (abricots, pêches, nèfles, etc.) uniquement en début de production, dans les marchés de gros au début du confinement alors que l'approvisionnement de ces marchés en fraise, produit très périssable, a été régulier selon l'ONAGRI. De ce fait, les répercussions sur les prix de ces produits ont été relativement modérées.

Pour l'élevage, certains agriculteurs interviewés nous ont signalé des difficultés pour la commercialisation de certains animaux : les taurillons à l'engraissement ou les génisses par exemple, suite notamment à la fermeture des marchés à bétail en début de confinement.

Globalement, les apports aux marchés de gros ont été suffisamment réguliers pour faire face à la demande des consommateurs. Ainsi, à titre d'exemple, les apports au marché de gros de Bir El Kassaâ qui fournit le Grand Tunis n'ont pas été fondamentalement bouleversés durant le confinement. Selon le site de l'ONAGRI, les apports ont été presque équivalents à ceux de la même période une année auparavant. Il en est de même pour les prix, sur ce même marché.

Au niveau macro-économique, les données sur les prix à la consommation des ménages de l'INS révèlent une tendance au ralentissement de l'inflation juste avant la crise et une légère remontée pendant celle-ci. Ainsi, le taux d'inflation passe de $5,8 \%$ à $6,3 \%$, en glissement annuel entre les mois de mai 2019 et mai 2020 (INS, 2020). L'alimentation ayant connu une inflation plus faible que la moyenne, $4,7 \%$, cela témoigne d'une certaine stabilité dans les rapports entre l'offre et la demande. La tendance à la baisse de l'inflation reprendra d'ailleurs son cours au mois de juin avec un taux d'inflation général de $4,8 \%$, et de seulement $4,3 \%$ pour l'alimentation (INS, 2020).

\section{3 Évolution de la demande en produits agricoles}

\subsection{Accroissement de la demande par constitution de stocks de sécurité et détournement de certains produits}

Des pénuries de produits alimentaires dans les commerces de détails ont été relevées par les médias avant et au début de la période de confinement. Elles s'expliquent par le comportement de sécurisation de l'approvisionnement familial des consommateurs, qui ont vite paniqué.

En effet, bien avant le confinement, la population a commencé à constituer des réserves de produits de première nécessité pouvant facilement être stockés: semoule, pâtes, farine, lait UHT, huile d'olive et de graines, riz, sucre, produits traditionnels, etc. Ce comportement s'est traduit par une pression forte sur les circuits d'approvisionnement, même si nous n'avons pas observé de rayons vides dans les grandes surfaces ou chez les épiciers de quartiers.

Le détournement de certains de ces produits fortement subventionnés, destinés à l'alimentation humaine (blé dur, orge), pour l'alimentation du bétail et la prolifération d'ateliers de fabrication de pain traditionnel en dehors des circuits officiels ont augmenté la pression sur ces produits de première nécessité.

Le problème d'accès alimentaire s'est surtout posé pour les populations rurales dont l'approvisionnement dépend de circuits de distribution plus fragiles du fait de leur éloignement des grands marchés et des grandes entreprises agroalimentaires, et de la petite taille des structures de commercialisation de détail (déclaration du ministre du Commerce le 16 avril).

Les populations rurales, ayant perdu leurs habitudes ancestrales de constitution de stocks de céréales et de légumineuses pour l'autoconsommation, se sont trouvées les plus démunies face aux perturbations induites par le confinement, telles que les restrictions de circulation, la fermeture des marchés hebdomadaires (les souks) et la tension sur certains produits alimentaires. En effet, selon l'INS, le niveau d'autoconsommation en milieu rural n'est plus que de $1,2 \%$ pour les céréales, de $0,5 \%$ pour les légumineuses et de 
$14,4 \%$ pour la viande; au plan national, le niveau d'autoconsommation des céréales a baissé de 9,9\% en 1980 à $0,7 \%$ en 2010 et pour les légumineuses, de 5,3 à $0,3 \%$ pour la même période (INS, 2013).

La réponse des pouvoirs publics a été l'injection de quantités plus importantes qu'à l'accoutumée de produits de base et notamment les semoules et la farine. Ainsi, selon le ministère du Commerce, les quantités journalières de blé livrées aux meuneries par l'Office des céréales sont passées de 12000 quintaux par jour avant la crise à 16000 durant le confinement (Karray et al., 2020).

\subsection{Baisse du revenu des ménages et impact sur la demande alimentaire}

En l'absence de dispositif de suivi de l'évolution des revenus par catégorie socioprofessionnelle, il est difficile de présenter une estimation fiable de l'impact de la Covid-19 et du confinement sur le revenu des ménages en général et de celui des agriculteurs et des ruraux en particulier. Au moment de la rédaction de cet article (juillet 2020), nous disposons néanmoins de deux projections de l'impact du confinement sur l'économie et le revenu des ménages.

La première, réalisée par le Forum tunisien des droits économiques et sociaux - FTDES (Mahjoub, 2020), montre, en se basant sur le taux de pauvreté avant la crise, que l'impact de celle-ci, selon une hypothèse de baisse du PIB de $5 \%$ (inférieure à celle annoncé par le chef du gouvernement) serait une augmentation du nombre de pauvres de 36,2\% en 2020. Cela correspondrait au passage du taux de pauvreté de 15,2 à $20,7 \%$ de la population totale, soit le niveau de la pauvreté constaté il y a dix ans. L'étude ne donne toutefois pas plus de précision sur la répartition de cette augmentation par catégorie socioprofessionnelle et par milieu de résidence.

De son côté, l'étude menée par l'IFPRI et l'ITCEQ (Elkadhi et al., 2020) montre que la durée du confinement est le facteur primordial expliquant l'ampleur probable de l'impact négatif de la crise. Ainsi, dans l'hypothèse d'une durée de confinement de trois mois, le secteur agricole tunisien perdrait 19500 emplois, soit $-0,1 \%$, contre une perte totale de 430000 emplois pour l'ensemble de l'économie, soit $-12,2 \%$. Du point de vue du produit intérieur brut, l'impact serait de l'ordre de $-11,6 \%$ pour l'ensemble de l'économie, alors que le secteur agricole ne connaîtrait que $-0,1 \%$ de perte. De même, en termes de revenu, la perte pour les employés du secteur agricole et les ruraux en général serait inférieure à la moyenne nationale tous secteurs confondus (Elkadhi et al., 2020). Ces données montrent bien que le secteur agricole est mieux outillé pour résister à la crise, mais elles ne tiennent pas compte des ruraux actifs en dehors du secteur agricole et des agriculteurs pluriactifs qui risquent de perdre une part importante de leur revenu.

La perte du pouvoir d'achat des consommateurs concerne d'abord les populations non agricoles qui, de ce fait, ont vu leur capacité à accéder à l'alimentation se réduire. Pour faire face à cette situation et aux risques sociaux et nutritionnels que cela pouvait entraîner pour les catégories de population les plus précaires, le gouvernement a mis en place un filet social avec l'octroi d'une prime de $200 \mathrm{DT}$ (66 euros) par mois de confinement pour environ 623000 ménages à faible revenu (perte d'emploi du chef de ménage ou chômage forcé du fait du confinement). Cette prime a concerné principalement les centres urbains, du fait que cette catégorie de population est mieux repérable dans cette situation qu'en milieu rural.

Bien que le secteur agricole, au niveau national, ait été globalement peu affecté par la crise sanitaire en termes de perte de revenu, il faut considérer qu'il existe une grande diversité de situations agricoles et rurales de ce point de vue. Ainsi, dans les régions rurales marginales avec des populations en forte précarité, les effets de la crise ont été les plus sévères. En effet, dans ces zones, l'agriculture ne permet pas d'assurer un revenu suffisant pour les populations précaires dont la vie dépend des activités non agricoles (très perturbées par la crise Covid-19) et des transferts d'argent depuis les zones urbaines et l'étranger.

Mais ce sont surtout les ruraux transformateurs de produits agricoles (fromageries traditionnelles, fabricants traditionnels d'harissa, de couscous, de préparations à base de farines de céréales et de légumineuses), de produits forestiers non ligneux (huiles essentielles de lentisque, romarin, myrte...), de miel et les fabricants de produits artisanaux (poteries, etc.) commercialisés sur les bords des routes ou dans les foires régionales et nationales, qui ont vu leurs ventes, et donc leurs revenus, chuter (témoignage des artisanes de Sejnane dans le nord de la Tunisie - voir le reportage sur le site Anadolu Ajansi : https:// www.aa.com.tr/fr/afrique/covid-19-bizerte-la-campagne-tuni sienne-doublement-1\%C3\%A9s\%C3\%A9e/1797761 [2020/ 06/29]). Il en est de même des activités de tourisme rural (gîtes ruraux et tables d'hôtes) dans les zones éloignées, qui ont souffert de l'interdiction des déplacements entre les régions.

Ces répercussions sont d'autant plus importantes que, dans ces zones marginales, une proportion élevée de la population rurale vit à la fois de l'agriculture et d'activités complémentaires (artisanat, transformation des produits agricoles, etc.). Ces activités, bien que fragiles, s'étaient bien développées grâce à l'appui des projets de développement rural et territorial ces dernières années (Elloumi, 2019).

Les conséquences de la baisse du revenu de ces populations rurales pauvres sont doubles: d'une part, elles ont eu tendance à consommer moins de produits alimentaires; d'autre part, elles ont consommé en priorité des produits alimentaires de première nécessité (semoule, farine, sucre, huile de graine, lait, etc.) et d'origine locale.

Ainsi, en plein confinement, les interviewés du panel de suivi de l'INS déclaraient avoir peur de manquer de nourriture (les interviews ayant été réalisés du 29 avril au 8 mai 2020). La fréquence de cette opinion était plus élevée en milieu rural et dans les populations les plus pauvres. Par ailleurs, la même étude relevait que: «plus d'un tiers des ménages les plus pauvres ont réduit la quantité ou la qualité des biens alimentaires consommés durant le confinement total... Toutefois, moins de $1 \%$ des interviewés déclarent être restés sans manger pendant une journée entière par manque d'argent ou d'autres ressources » (INS et Banque mondiale, 2020a). À signaler que la seconde vague d'interviews (15-21 mai 2020) portant sur le même panel, a relevé une réduction de consommation alimentaire plus faible (INS et Banque mondiale, 2020a, 2020b).

\subsection{La réduction de la demande sur les marchés internationaux}

Pour les produits d'exportation phares (les dattes, l'huile d'olive et les produits de la mer), l'arrêt de l'économie mondiale du fait de la pandémie a fortement limité les 
possibilités de la Tunisie de commercer avec les pays étrangers habituels, mais à des degrés divers selon les produits.

Pour les dattes, la Tunisie bénéficiait d'une situation privilégiée sur certains marchés, notamment le Maroc, l'Union européenne et les États-Unis d'Amérique, du fait de son savoir-faire en matière de production et de commercialisation. Malgré une très bonne récolte fin 2019 et des perspectives d'exportation prometteuses, la crise sanitaire a donné un coup d'arrêt aux exportations tunisiennes (baisse de $43 \%$ des quantités et de $46 \%$ en valeur pour le mois d'avril). De plus, cette crise a coïncidé avec le mois du Ramadan (24 avril au 23 mai) qui connaît habituellement une consommation importante de dattes au niveau mondial. Ce pic de consommation ne pourra pas être rattrapé dans les mois suivants, ce qui plonge la filière tunisienne dans une crise sans précédent. En effet, cette baisse de la demande extérieure s'est traduite par une baisse d'environ $10 \%$ des quantités exportées sur les cinq premiers mois de 2020, engendrant une perte de plus de $12 \%$ en valeur par rapport à l'année 2019. Pour certains marchés, la baisse a été beaucoup plus forte: Maroc, $-26 \%$, et États-Unis, $-64 \%$, en volume

Pour les produits de la mer et de l'aquaculture, la fermeture temporaire de certains marchés, notamment ceux de l'Italie, de la Libye et de l'Espagne (55\% des quantités exportées), a entraîné une baisse importante des quantités exportées et des revenus des acteurs de cette filière.

Pour l'huile d'olive, la récolte record d'olives en 2019 2020 devait permettre à la Tunisie d'exporter environ 250000 tonnes d'huile, représentant pour le pays une entrée de devises équivalant à environ 2000 millions de dinars tunisiens (DT). La crise sanitaire n'a pas eu un effet sur les quantités exportées, qui ont connu une nette amélioration pour atteindre 232000 tonnes contre moins de 100000 tonnes une année auparavant; mais les recettes en devises sont loin des attentes des acteurs de la filière et n'avaient, à la fin du mois de mai, atteint que 1400 millions de DT. Cette situation s'explique principalement par la situation du marché international qui connaît un excédent d'offre suite à la succession de deux récoltes exceptionnelles, en 2018/2019 et 2019/2020.

Enfin, d'autres filières moins importantes en termes de volume d'exportation ont connu des perturbations de même nature: la filière pomme de terre a connu des difficultés d'exportation notamment sur le marché libyen (ONAGRI, 2020a), celle des fruits à noyau (pêche, prune...) a connu une baisse des quantités exportées de $22 \%$ entre janvier et début juin 2020, par rapport à la même période en 2019 (ONAGRI, 2020a).

\section{Quelles leçons tirer de la crise Covid-19?}

Selon les constats réalisés, nous pouvons estimer aujourd'hui que le gouvernement a réussi à faire face à la pandémie avec le moins de dégâts possible: au plan sanitaire, selon un communiqué du ministère de la Santé en date du 28 juin 2020, la Tunisie a maîtrisé la Covid-19, avec seulement 1172 contaminations pour une population totale d'environ 12 millions d'habitants, avec 1029 guérisons, 50 décès et 93 cas qui sont encore porteurs du virus; à cela s'ajoute le fait que les rares cas détectés depuis près d'un mois sont des cas importés découverts chez des Tunisiens ou des étrangers venant d'autres pays. Au plan économique, les risques de famine ont été évités et il n'y a pas eu de crises sociales issues de la crise sanitaire et du long confinement imposé et globalement respecté par la population. L'INS observe même une amélioration du moral des citoyens constituant le panel entre la première et la seconde vague d'interviews (fin avril et mi-mai) quant aux risques de perte de revenu et aux difficultés d'accès aux produits alimentaires (INS et Banque mondiale, 2020a, 2020b).

Sur le plan économique les résultats sont moins évidents à estimer à ce stade : la baisse du PIB annuel de la Tunisie pour 2020 est estimée entre $6,5 \%$ selon la primature et $5 \%$ selon l'étude du FTDES (Mahjoub, 2020). Certaines analyses affirment que le secteur agricole a été plus résilient que les autres secteurs de l'économie (Elkadhi et al., 2020).

Il faut reconnaître que si ces résultats sont liés aux mesures prises par le gouvernement, ils sont aussi le fait d'une conjoncture favorable comme la constitution de stocks de produits alimentaires de grande consommation en prévision du mois de Ramadan (24 avril au 23 mai). Ces stocks sont constitués annuellement par les différents opérateurs des filières sous la supervision du ministère du Commerce et des autres ministères concernés. Par ailleurs, la période observée est celle de la pleine saison de production de certains fruits et légumes, ce qui a permis d'assurer une offre importante en produits frais malgré quelques difficultés de transport et de mise en marché. De plus, les prix des principaux produits alimentaires importés par la Tunisie (céréales et produits oléagineux) n'ont pas augmenté notablement, malgré les menaces de certains pays exportateurs de limiter leurs exportations.

Par ailleurs, le niveau de l'offre alimentaire par l'agriculture tunisienne était élevé du fait de récoltes exceptionnelles pour trois produits en 2019/2020 par rapport à la campagne précédente: huile d'olive (350000 tonnes contre 140000 tonnes), céréales ( 24 millions de quintaux contre 17 millions) et dattes (avec une production de 340000 tonnes en hausse de $18 \%$ ). Malheureusement, les difficultés rencontrées sur les marchés internationaux n'ont pas permis de réaliser les performances économiques attendues et la balance commerciale a affiché un solde négatif au terme des cinq premiers mois de 2020 (Elloumi, 2020).

\subsection{L'importance du rôle de la production nationale dans la sécurité alimentaire}

Avant la pandémie, la Tunisie affichait un niveau de couverture de ses besoins alimentaires par la production nationale d'environ $60 \%$ en termes de calories (de LattreGasquet et al., 2017). La Tunisie est quasiment autosuffisante en fruits, légumes et viande de volaille, et affiche des taux de couverture de plus de $90 \%$ pour les viandes rouges et le lait. Par contre, ce taux est seulement de $40 \%$ pour les céréales, y compris celles destinées à l'alimentation animale. Pour les huiles, malgré les récents records de production et le classement de la Tunisie parmi les premiers producteurs d'huile d'olive au monde, la consommation nationale de matières grasses est dominée par les huiles de graines importées, la production d'huile d'olive étant majoritairement destinée à l'exportation. 
Durant la période de confinement, la production nationale a joué un rôle important dans la couverture de la demande. À l'exception des produits issus des céréales (farine, semoule), l'approvisionnement des centres de consommation a été assuré de manière régulière et suffisante par la production locale. Ceci dénote une bonne capacité d'adaptation des producteurs et des acteurs de l'aval en situation de crise pour répondre à la demande des consommateurs. Mais c'est aussi le résultat d'un contrôle administratif assez drastique assuré par le ministère du Commerce qui encadre la constitution de stocks régulateurs dont la gestion est assurée conjointement par l'Office du commerce et les acteurs de chaque filière: groupement interprofessionnel pour les produits de l'aviculture, société (publique) El Louhoum pour la viande rouge, industriels pour le lait et les produits laitiers, Office des céréales en collaboration avec les minotiers pour les céréales.

Pour les produits céréaliers, et malgré des stocks suffisants, le gouvernement a passé en urgence des commandes sur le marché international avec livraison au mois de mai afin d'assurer largement la couverture des besoins jusqu'à la récolte (juin-juillet). En dépit de cela, des perturbations dans l'approvisionnement ont été observées, qui sont surtout le résultat des difficultés de fonctionnement des circuits de distribution en période de crise, de comportements de panique des consommateurs et de l'apparition de processus spéculatif pour certains produits (farine et semoule principalement).

\subsection{Le rôle du marché national dans l'absorption de la production}

Inversement, la crise Covid-19 a montré le rôle primordial du marché domestique pour l'absorption de la production. Ainsi, malgré le risque de propagation de la pandémie, tous les acteurs se sont mobilisés pour assurer l'écoulement de la production, sa transformation éventuelle et son acheminement vers les lieux de consommation. Selon nos enquêtes, les acteurs de la filière lait se sont mobilisés de manière assez efficace pour assurer la collecte, la transformation et le stockage de la production, particulièrement périssable. Cette mobilisation a permis de faire face à la demande tout au long du confinement et notamment durant le mois du Ramadan qui constitue habituellement un pic de consommation. La mobilisation des industriels du lait a été totale puisque malgré le pic de production du printemps, aucun problème de collecte n'a été signalé. Cela a été par ailleurs confirmé par nos enquêtes auprès de producteurs à Mateur, Sfax et dans le Grand Tunis.

À l'inverse, pour d'autres produits, la consommation nationale n'a pas été suffisante pour absorber la totalité de la production. C'est le cas, par exemple, des pommes de terre ou de certains fruits de saison, notamment les pêches. Pour ces derniers, c'est le caractère périssable de la production, qui connaît depuis un certain nombre d'années une surproduction cyclique, qui pose problème puisque la transformation n'a pas décollé. Par contre, pour les pommes de terre, ce qui a posé problème est la baisse de la consommation (fermeture de la restauration collective et absence de touristes), à laquelle s'est ajoutée la baisse de l'exportation vers la Lybie.

\subsection{Les effets indirects de la crise}

Les observations faites tout au long du confinement à travers les médias et les réseaux sociaux, nous ont permis de voir émerger de nouveaux comportements d'achat et d'alimentation.

Ainsi, en termes d'achat, et mise à part la constitution de stocks en début de crise pour faire face à l'incertitude de l'approvisionnement alimentaire à venir, nous avons pu voir se consolider la préférence de certains consommateurs pour l'approvisionnement sur les marchés locaux avec des filières courtes, préférence qui progressait déjà avant la crise sanitaire.

Ces phénomènes émergents ont été consolidés par le recours à l'e-commerce qui a connu un grand développement, même s'il reste difficile à chiffrer pour le moment. Ainsi, plusieurs plateformes de vente sur Internet et de livraison à domicile de produits alimentaires ont été mises en place et ont vu leur chiffre d'affaires croître durant les premières semaines de confinement. Ces plateformes ont même eu du mal à faire face à la demande du fait des difficultés de recrutement de personnel pour faire fonctionner les plateformes ou des difficultés à contractualiser et à s'approvisionner auprès de producteurs, peu coutumiers de ce mode de commercialisation, ou encore des difficultés de livraison du fait du couvre-feu. Selon nos enquêtes, ces plateformes de e-commerce ont permis à certains producteurs d'obtenir de nouveaux débouchés et ainsi de maintenir leurs activités de production et un chiffre d'affaires acceptable. Reste à savoir si ces nouvelles pratiques de commercialisation et d'achat de produits alimentaires locaux vont pouvoir durer, voire se consolider après la crise Covid-19.

\section{Conclusion : l'agriculture post-Covid-19, une occasion de dépassement de la crise du secteur agricole?}

L'agriculture tunisienne semble, au stade actuel de la pandémie, avoir montré une capacité de résilience certaine face à la crise sanitaire. Toutefois, cette résilience correspond surtout au fait que l'approvisionnement en fruits et légumes, en viande de volaille et en produits laitiers a pu se poursuivre à peu près normalement malgré le confinement. Cependant, la question centrale de la dépendance de la Tunisie aux marchés extérieurs pour certains produits alimentaires demeure et ne semble pas être mise en débat par les autorités à l'heure actuelle. Cela concerne les céréales, les huiles de graines et le sucre pour l'alimentation humaine, les céréales secondaires et le tourteau de soja pour la production de la viande de volaille, de la viande bovine et du lait: au final, plus de $40 \%$ des calories consommées sont importées (de Lattre-Gasquet et al., 2017)

Cette dépendance montre la fragilité de la sécurité alimentaire, basée sur les échanges internationaux, face aux crises politiques et sanitaires et surtout face au changement climatique qui risque d'aggraver la dépendance alimentaire de la Tunisie dans un futur proche.

La crise sanitaire et le confinement de la population ayant entraîné un arrêt quasi total de l'économie ont agi comme révélateurs des limites des modèles économique et technique de développement du secteur agricole tunisien. D'ailleurs, lors 
de notre enquête, les interviewés, simples agriculteurs ou responsables professionnels, ont tous pointé du doigt les limites du modèle actuel et appelé de leurs vœux un changement de paradigme.

Or, pour dépasser cette crise structurelle du système alimentaire et construire un nouveau paradigme de développement qui permette d'ouvrir de nouvelles perspectives pour une réelle sécurité alimentaire, il faudrait agir à la fois sur le contenu technique du modèle, sur les choix économiques d'insertion dans la division internationale de la production agricole, et construire de nouveaux rapports entre le secteur agricole, les agriculteurs et les ruraux, d'une part, et le reste de la société dont les consommateurs urbains, d'autre part.

Ainsi, sur le plan technique, il est urgent pour des pays comme la Tunisie qui font déjà face aux effets du réchauffement climatique, de construire un nouveau modèle technique qui concilie l'augmentation de la production et un usage rationnel des ressources naturelles. Les prémices de ce modèle existent dans ce qui est communément appelé "l'intensification écologique» (Griffon, 2013). C'est à la recherche agronomique d'en développer les composantes techniques et les mesures d'accompagnement pour en faciliter l'adoption par les agriculteurs tunisiens.

Sur le plan économique, il n'est pas envisageable pour un pays comme la Tunisie de se couper du monde, mais la question est de trouver un équilibre durable entre insertion dans les marchés mondiaux et souveraineté alimentaire.

Enfin, pour les rapports entre les consommateurs et les producteurs, la crise Covid-19 a montré l'importance de l'agriculture locale et de sa capacité à se mobiliser pour répondre à la demande des consommateurs. Il est donc important de capitaliser sur ces acquis pour construire de nouveaux rapports plus équilibrés et bénéfiques à tous en termes de qualité des produits pour les consommateurs et de conditions de vie pour les ruraux. Les populations urbaines majoritaires devraient accepter de payer un peu plus cher les produits agricoles tunisiens, en mettant aussi dans la balance le coût sanitaire de la «malbouffe» qui caractérise leur régime alimentaire actuel.

\section{Références}

Elkadhi Z, Elsabbagh D, Frija A, Lakoud T, Wiebelt M, Breisinger C. 2020. The impact of Covid-19 on Tunisia's economy, Agri-food System, and Households. Middle East and North Africa: IFPRI, 13 p. DOI: $10.2499 /$ p15738coll2.133737.
Elloumi M. 2019. Le développement territorial comme alternative pour les régions marginales en Tunisie, entre mythe et réalité. In : Aderghal M, Genin D, Hanafi A, Landel P-A, Michon G, eds. L'émergence des spécificités locales dans les arrière-pays méditerranéens. Les impromptus du LPED, $\mathrm{n}^{\circ} 5$, chapitre 7 . Paris (France): LPED, pp. 367-383.

Elloumi M. 2020. La faillite des politiques agricoles et commerciales en Tunisie. [2020/02]. http://kapitalis.com/tunisie/2020/02/16/lafaillite-des-politiques-agricoles-et-commerciales-en-tunisie/.

FAO. 2020a. Marchés agroalimentaires et politiques commerciale au temps de la Covid-19. Rome (Italie): FAO, 6 p.

FAO. 2020b. Les répercussions de la Covid-19 sur l'accès des petits producteurs aux marchés. Rome (Italie): FAO, 10 p.

Griffon M. 2013. Vers une septième révolution verte. Revue Projet 2013/1(332): 11-19. DOI: 10.3917/pro.332.0011.

INS. 2013. Enquête nationale sur les dépenses, la consommation et le niveau de vie des ménages de 2010, volume $2:$ la nutrition. Tunis (Tunisie): INS, 99 p. + annexes.

INS. 2017. Enquête nationale sur les dépenses, la consommation et le niveau de vie des ménages de 2015, volume 1 : niveau des dépenses. Tunis (Tunisie): INS, $139 \mathrm{p}+$ annexes.

INS. 2020. Indice des prix à la consommation, base 100 en 2015 , communiqué de presse, mai 2020. [2020/07/06]. http://www.ins.tn/ $\mathrm{fr} /$ publication/indice-mensuel-des-prix-\%C3\%A0-la-consomma tion-familiale-ipc-juin-2020.

INS, Banque mondiale. 2020a. Suivi de l'impact socio-économique du Covid-19 sur les ménages tunisiens. Analyse des données de la $1^{\text {re }}$ vague (29 avril 2020-8 mai 2020). Tunis (Tunisie): INS, $11 \mathrm{p}$.

INS, Banque mondiale. 2020b. Suivi de l'impact socio-économique du Covid-19 sur les ménages tunisiens. Résultats de la $2^{\mathrm{e}}$ vague (15 mai 2020-21 mai 2020). Tunis (Tunisie): INS, 15 p.

Karray B, Boudiche S, Ayadi MA, Agrebi N, Skandrani Y. 2020. Mesures préservant la filière agricole et le bon fonctionnement de l'industrie agroalimentaire, face à la pandémie du Covid-19. Carthage (Tunisie): ITES, $76 \mathrm{p}$.

de Lattre-Gasquet M, Moreau C, Elloumi M, Ben Becher L. 2017. Vers un scénario « des usages agro-écologiques des terres pour une alimentation diversifiée et de qualité et un système alimentaire territorialisé » en Tunisie en 2050. OCL 24(3): 20 p. DOI: 10.1051/ ocl $/ 2017025$.

Mahjoub A. 2020. Pandémie Covid-19 en Tunisie : les inégalités, les vulnérabilités à la pauvreté at au chômage. Tunisie: FTDES, 35 p.

ONAGRI. 2020a. Évolution des exportations des produits agroalimentaires vers la Libye durant les quatre premiers mois de l'année 2020. Tunis (Tunisie): ONAGRI, 2 p.

ONAGRI. 2020b. Impact des répercussions de la crise Covid-19 sur la production, l'approvisionnement, les prix et les exportations des produits agricoles. La Lettre de l'ONAGRI 6(2): 49 p.

Citation de l'article : Elloumi M. 2020. L'agriculture tunisienne face à la Covid-19: impacts de la crise sanitaire et perspectives pour une agriculture résiliente. Cah. Agric. 29: 35. 